\title{
The TRANAIR rectangular grid approach to solving the nonlinear full-potential equation about complex configurations
}

\author{
FORRESTER T JOHNSON \\ The Boeing Company, P O Box 3707, Seattle, Washington 98124-2207, \\ USA
}

MS received 22 January 1991

\begin{abstract}
This paper describes TRANAIR, a numerical method that uses a rectangular grid to solve the nonlinear full-potential equation about general complex configurations. The grid is locally refined to resolve the high velocity gradients arising from leading edge expansions or shock waves. The grid penetrates the boundary (described by networks of quadrilateral panels) and is generated automatically. Discrete operators are constructed using the finite element method. The system of nonlinear discrete equations is solved iteratively using an orthogonal conjugate gradient method preconditioned by an exterior Poisson solver and a direct sparse solver. The primary emphasis of this work is to provide design engineers with an aerodynamics analysis tool (the TRANAIR code) which is as accurate, reliable, economical, and flexible to use as panel methods. Results obtained by using TRANAIR to analyse several interesting configurations are presented.
\end{abstract}

Keywords. TRANAIR rectangular grid approach; nonlinear full-potential equation; complex configuration.

\section{Introduction}

This paper describes the mathematical approach behind the TRANAIR rectangular grid computer code and then discusses the application of this code to the aerodynamic analysis of several configurations of varying complexity. TRANAIR was developed to replace panel codes as a workhorse aerodynamics analysis tool for full configuration design. TRANAIR contains the same configuration and boundary condition capability as most panel codes but solves the nonlinear, full-potential equation, allowing regions of differing total temperature and pressure to simulate propulsion effects. For the last twenty years, complex, full-aircraft configurations have been routinely analysed by panel methods. Panel-method users now take for granted the ability to add, move, or delete components at will; readily select and change boundary condition types; and obtain accurate solutions at reasonable cost in a day or two. 
The primary drawback of panel methods is their limitation to linearized supersonic or subsonic flows. Unfortunately, the analysis of nonlinear effects requires computations in the volume exterior to the configuration surface. In the past, generation of a grid on which to make these computations has been anything but routine. Moreover, many current flow solver algorithms have difficulty handling grids about complex configurations due to their size and topological anomalies.

TRANAIR was developed to eliminate these problems. It circumvents the issues involved in surface-fitted grid generation by employing a uniform rectangular grid together with local grid refinement. The use of a rectangular grid allows the application of powerful preconditioners to ensure convergence. Reliability, generality, efficiency, and usability of TRANAIR approach those of panet codes. In fact, the input deck is virtually identical to that of a panel code in use for the past ten years (Johnson 1979). Run times are usually comparable to those for panel codes even though the flow now contains shocks and other nonlinear effects. In order to achieve these results, a wide variety of numerical algorithms have been combined to form a rather nonstandard method, as described in the next section. (Others have also successfully employed rectangular grids to solve nonlinear flow problems, e.g. Wedan \& South 1983; Desai et al 1987).

\section{Description of the method}

This section describes the numerical method. First, the continuous problem to be solved is presented. Then, the grid and boundary are described, and finally the construction of the discrete operator system and its iterative solution are explained.

\subsection{Problem definition}

The full potential equation is

$$
\mathscr{L}(\Phi) \equiv \nabla \cdot \rho \nabla \Phi=0
$$

where the density is given by

$$
\rho=\rho_{\infty}\left\{1+\frac{1}{2}(\gamma-1) M_{\infty}^{2}\left[1-\left(q^{2} / q_{\infty}^{2}\right)\right]\right\}^{\gamma /(\gamma-1)} .
$$

Here, $\Phi$ is the total velocity potential to be determined, $q=\|\nabla \Phi\|_{2}$ is the speed, $M$ is the Mach number, $\gamma$ is the ratio of specific heats, and the subscript $\infty$ denotes value at a far upstream location.

The far-field condition is that the perturbation potential $\phi=\Phi-\Phi_{\infty}=O(1 / R)$. The normal flow boundary condition may be given by $\rho(\partial \Phi / \partial n)=h$, where $h$ is zero for impermeability and may be specified nonzero on other surfaces, such as engine inlets. We may impose the Dirichlet condition $\Phi=g$ on an engine exhaust surface, where tangential flow can be prohibited by specifying $g$ to be constant. The boundary conditions on wakes represent conservation of mass and normal momentum across the wake via

$$
\hat{n} \cdot \Delta(\rho \nabla \Phi)=0, \Delta p=0,
$$


where $\Delta$ represents the jump across the wake surface and

$$
p=p_{\infty}\left\{1+\frac{1}{2}(\gamma-1) M_{\infty}^{2}\left[1-\left(q^{2} / q_{\infty}^{2}\right)\right]\right\}^{\gamma /(\gamma-1)} .
$$

Linearization of $\Delta p$ about $p_{\infty}$ assuming shall $\nabla \phi$ leads to the equivalent Dirichlet condition that $\Delta \Phi$ is constant along the wake in the direction of $\mathbf{V}_{\infty}$. The circulation $\mu$ at the trailing edge is determined by a Kutta condition imposed there.

The boundary value problem described above can be cast in variational form for the purpose of applying a finite element method of solution. The principle we use (which is a generalization of the Bateman principle, Bateman 1930), states that the functional

$$
\begin{aligned}
J= & \int_{\text {flow }} p \mathrm{~d} V+\int_{\text {inlets }} h \Phi \mathrm{d} S-\int_{\text {wakes }} \alpha \rho\left(\frac{\partial \Phi}{\partial n}\right)(\Delta \Phi-\mu)+ \\
& +\frac{\alpha \rho}{2 \Delta 1}(\Delta \Phi-\mu)^{2} \mathrm{~d} S+\int_{\text {exits }} \rho \frac{\partial \Phi}{\partial n}(\Phi-g)-\frac{\rho}{2 \Delta 1}(\Phi-g)^{2} \mathrm{~d} S,
\end{aligned}
$$

is stationary. Here an $\alpha$ denotes average value.

A modification of the above formulation allows the simulation of flows involving regions of differing total temperature and pressure. Potential flow exists in each separate region as long as total temperature and pressure are constant in that region, but pressure and density must be redefined in the following way:

$$
\begin{aligned}
& p=p_{\infty} r_{p}\left\{1+\frac{1}{2}(\gamma-1) M_{\infty}^{2}\left[1-\left(q^{2} / q_{\infty}^{2} r_{T}\right)\right]\right\}^{\gamma /(\gamma-1)}, \\
& \rho=\rho_{\infty}\left(r_{p} / r_{T}\right)\left\{1+\frac{1}{2}(\gamma-1) M_{\infty}^{2}\left[1-\left(q^{2} / q_{\infty}^{2} r_{T}\right)\right]\right\}^{\gamma /(\gamma-1)} .
\end{aligned}
$$

Here, $r_{p}$ is the ratio of the total pressure in the region to the freestream total pressure and $r_{T}$ is the ratio of total temperature in the region to freestream total temperature. The regions are assumed to be separated by fixed wake surfaces on which two jump boundary conditions similar to those stated before are applied.

\subsection{Surface geometry and grid specification}

The boundary surfaces are prescribed via networks of panels. This input format allows relatively easy specification of complicated boundaries and is identical to the input format of panel method codes that are extensively used for linear flow analysis (Johnson 1979).

The surface networks are embedded in a rectangular grid confined to a finite rectangular computational box. The restriction of the grid to a compact region is justified through a source transformation. The argument goes as follows. Suppose that the partial differential operator $\mathscr{L}$ is well approximated by a constant coefficient differential operator $\mathscr{T}$ in the far field. Then the original differential equation $\mathscr{L} \Phi=0$ is equivalent to

$$
\mathscr{2}+(\mathscr{L}-\mathscr{T}) \mathscr{G} * \mathscr{Q}=0,
$$

where $\mathscr{G}$ is the Green's function for $\mathscr{T}$ such that $\mathscr{T}(\mathscr{G} * \mathscr{Q})=\mathscr{Q}$ for all $\mathscr{Q}$ and $\Phi=\mathscr{G} * \mathscr{Q}$ satisfies the proper far-field condition. If we use the sources 2 as the unknowns, then we need restrict our computation to only that region where $\mathscr{Q}$ is nonzero. We note that for most problems of interest, $\mathscr{Q}$ approaches zero at $\infty$ much faster than $\Phi$ 
approaches $\Phi_{\infty}$. Hence, it is possible to find a finite computational box outside which the approximation $\mathscr{Q} \cong 0$ holds very well. For the full-potential equation, the Prandtl-Glauert operator may be taken as $\mathscr{T}$ and for a wing in transonic flow the computational box needs to extend only one or two chord lengths away. Construction of a Green's function for the Prandtl-Glauert operator, which satisfies the far-field boundary condition for the original problem, is relatively straightforward. This is, in fact, facilitated by the use of a rectangular grid (Buneman 1971; Rubbert et al 1986, p. 49; TRANAIR 1987) provided the density of the uniformly refined rectangular grid is sufficiently fine.

For a given computational box, the process of constructing the volume grid is automatic and is described below. We start with a coarse uniform rectangular grid, called the global grid, that contains all boundary surfaces but is otherwise independent of the boundary surfaces. The global grid is locally refined, where necessary, in a hierarchical manner, i.e., selected grid boxes are refined into eight geometrically similar boxes. This process is repeated to give a grid with any desired local resolution.

The initial refinement is controlled by two criteria:

- The first criterion is based on the length scale of the surface panels used to describe the boundary. Every box element that is sufficiently close to a panel is refined if some weighted length scale associated with the panel is smaller than the length scale associated with that box element. This criterion is effective in providing denser grid near certain parts of the boundary if it is known that the solution there would have stronger gradients.

- The second criterion allows refinement away from boundary surfaces. Special "regions of interest or disinterest" can be prescribed each with desired minimum and maximum refinement levels. All the box elements in these special regions are refined recursively until the minimum level is reached. Further refinement depends on the first criterion. The special regions are hexahedral and provide a fair amount of flexibility in generating off-surface refinement. Such refinement is useful in problems where large gradients such as shock waves may exist.

We note that, although it is possible to control the grid initially based on the knowledge of the problem, it is necessary to provide for adaptive refinement based on solution errors. We are currently implementing adaptive refinement (or derefinement) in the code whereby dependence of the final solution on the initial criteria provided by the user will be significantly reduced or eliminated entirely.

The locally refined grid formed by the process described above is usually an unstructured collection of boxes. We have developed a compact data structure based on oct-trees (Samet 1984; Samant et al 1988) that contains essentially all the information regarding the refined grid. Asymptotically, this data structure requires storage equal to $10 / 7$ the number of unrefined boxes for the box information. The data structure allows efficient extraction of a variety of information, such as the location of nodes and element centroids, box size, box level, node indices, box adjacency and identity of boundary boxes.

The oct-tree data structure we have used is primarily based on boxes. At the beginning of the oct-tree array certain overhead information that describes the size of the grid is stored. This is followed by two blocks of data, each as long as the size of the global grid, describing its refinement. Then the branch data elements of the tree follow. These describe the hierarchical refinement and form the bulk of the oct-tree. A typical branch data element in an oct-tree is illustrated in figure 1. The 


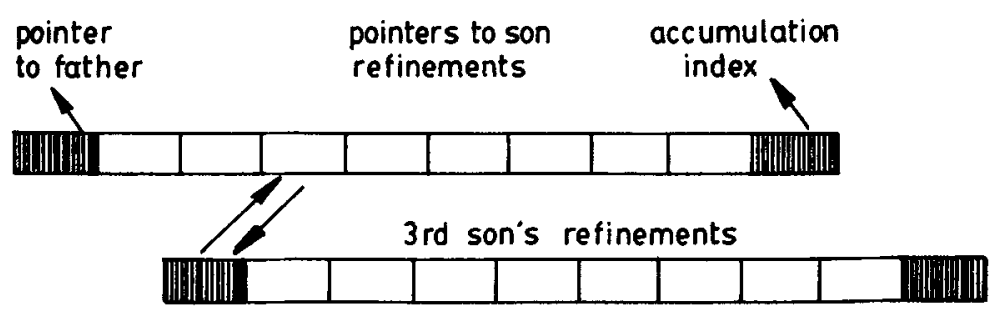

Figure 1. Oct-tree data structure element.

first word in a branch data element points to the father box, the next eight words point to the refinement branches of the sons, if any, and the last word contains an accumulation index specifying the number of nodes encountered up to that point in the oct-tree. A null pointer (value zero) in the son entry in any branch data element represents an unrefined box. Any unrefined box cut by a boundary is identified by a negative number (equal to its index in a list of such boxes) placed in the son entry in a branch data element. This is a convenient and compact way of accounting for the presence of the boundary.

We have extended the oct-tree data structure further to accommodate nodal information. Nodes are indexed by assigning the index of a box to the node at its lower-left-near corner. In order to account for all nodes at refinement interfaces we perform pseudo refinement (see figure 2). This allows us to keep track of the nodes as well as the boxes using the same oct-tree with only a modest increase in storage. Boxes added by the pseudo refinement are used only to identify nodes and are not finite elements.

Even though the oct-tree data structure described above is able to reflect an arbitary collection of hierarchically refined grid cells, it is convenient to restrict the refinement pattern. We require that no two face or edge neighbours in a "legal" refined grid differ by more than one level (see figure 2). This rule prevents pathologically large stencils under certain circumstances, but allows refinement down to an arbitrary level within one adjacent coarse grid box.

The location of a node or a centroid of an unrefined box can be calculated by descending to the ancestor in the global grid and then using the global box information. Adjacency or box-to-box connectivity information can be obtained by starting at a box and descending the oct-tree to the root of the branch that includes both boxes and then ascending a complementary path to the neighbouring box.

\subsection{Discretization}

The finite element method is used to discretize the partial differential operator. On each box element a standard trilinear basis function parameterized by eight unknowns

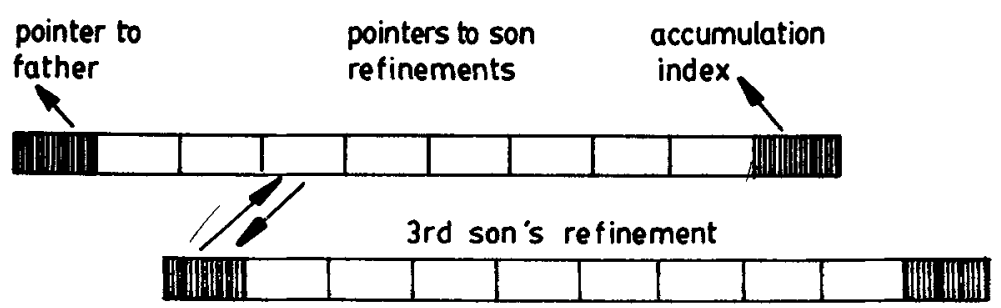

Figure 2. Some issues in hierachical refinement. 
(a)
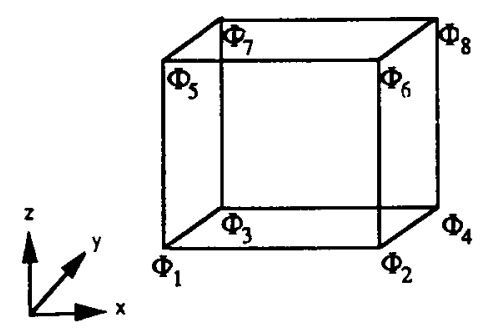

(b)

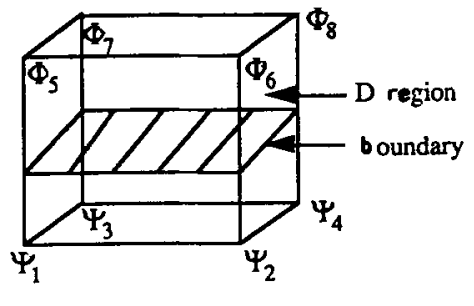

Figure 3. Box finite element with eight corner unknowns. (a) Standard box. (b) Boundary box.

located at each corner of the box element is defined. Element stiffness matrices are generated by taking variations of the functional $J$ in (5) with respect to each degree of freedom. For standard element not cut by a boundary we may write the variation by

$$
\delta J=-\int_{\text {flow }} \rho \nabla \Phi \cdot \nabla \delta \Phi \mathrm{d} V .
$$

In evaluating the integrals, the density is taken as a piecewise constant function over the element with the value of density at centroid. Since the boxes are obtained by hierarchical refinement, every standard element has the same element stiffness matrix up to a constant factor that depends only on the refinement level of the element and the density at the centroid of that element. This can be exploited to drastically reduce storage requirements. Only one element stiffness matrix must be stored for all boxes not cut by the boundary.

For the boxes cut by the boundary, the element stiffness matrices are derived from (5), including appropriate surface integral terms. The domain of volume integration referred to as a $D$ region, is any connected region in the box containing flow, see figure $3 \mathrm{~b}$. The domain for the surface integrals is the part of the boundary cut by the box. The trilinear approximation function is defined on boxes containing the boundary in the same manner as above; however, corner unknowns on the other side of a boundary surface can be viewed as extrapolated values and are denoted by $\Psi$. For complex geometries, the $D$ regions can take any shape, and the integration involved in computing element stiffness matrices appears to be a formidable task at first sight. But the integrands are polynomials, so that Gauss' and Stokes' theorems and simple one-dimensional integration formulas can be used to systematically reduce the integrals over a complicated volume to point evaluation at vertices of the bounding surface. It is possible to have more than one $D$ region and consequently more than one trial function in a given rectangular box and more than one unknown at a grid point. Each $D$ region has a distinct element stiffness matrix that must be stored. However, these elements represent typically only 10 to $20 \%$ of the elements needed to give an accurate solution of the boundary value problem. Hence, the storage required is acceptable.

In order to maintain conservation of mass across the grid interfaces in a finite element method, we must maintain the continuity of basis function. This is achieved through the introduction of psuedo unknowns, which lie on the edges and faces of boxes with finer neighbours, and equating them to be averages of their parents at the endpoints of the edges and the corners of the faces. 
In the full potential case, standard first-order upwinding of the density is used to produce the artificial viscosity required when supersonic flow is present (Hafez et al 1978). Such an upwinding is given by replacing $\rho$ in the full potential equation with

$$
\bar{\rho}=\rho-\mu \hat{V} \cdot \nabla_{-} \rho \text { with } \mu=1-M_{c}^{2} / M^{2},
$$

where $\hat{V}$ is the normalized local velocity, $\nabla_{-} \rho$ is an upwind difference, $M$ is the local Mach number, and $M_{c}$ is the cut-off Mach number. This upwinding is first-order accurate.

\subsection{Solution techniques}

Since the resultant discrete system is generally large, nonsymmetric, nonlinear and often poorly conditioned, we have chosen the GMRES algorithm (Wigton 1985; Saad \& Schultz 1986) as the basic iterative solver. GMRES is only effective, however, when it is preconditioned appropriately to produce a favourable distribution of eigenvalues. Denoting the solution unknown symbolically by $\chi$ let the discrete system to be solved be

$$
L(\chi)=f .
$$

The standard left preconditioning is the application of GMRES to

$$
N^{-1} L(\chi)=N^{-1} f
$$

where $N^{-1}$ is the preconditioner. The standard right preconditioning is the application of GMRES to

$$
L T^{-1}(Q)=f
$$

where $Q=T \chi$. We have found that a combination of right and left preconditioning works best for the types of problems being considered here. We use combinations such as

$$
v T N^{-1} L T^{-1} Q=v T N^{-1} f
$$

where $v$ is a cutoff imposed at the outer boundary of the computational box.

The right preconditioner, $T^{-1}$, is the convolution with the Green's function for the discrete Prandtl-Glauert operator defined on a uniform rectangular grid that is a subset of the entire grid. For other unknowns, this operator is simply identity. Such a preconditioner is extremely effective for subsonic regions and ensures that the far-field boundary condition is satisfied. Both the application of $T$ and the preconstructed Green's function $T^{-1}$ are highly vectorizable operations.

The left preconditioner, $N^{-1}$, is chosen to be an inverse of the global stiffness matrix restricted to a reduced set. The reduced set consists of all unknowns that are located at corners of boundary boxes (all unknowns associated with all the $D$ regions in every boundary box are included), the refined boxes, and those boxes where the flow is supersonic. Special boundary conditions are applied at closure unknowns to close the system. It is quite feasible to perform a direct sparse incomplete factorization of $N$ for the following reasons.

(1) A drop tolerance can be introduced into the sparse elimination process allowing 
small elements in the decomposition to be dropped as they are generated. This has a cascading effect and dramatically reduces fill (Bussoletti 1988).

(2) A grid-based, nested dissection ordering can be generated for this reduced set so that the fill is reduced during elimination.

(3) The reduced set is often significantly smaller than the total number of degrees of freedom in the problem.

To handle nonlinearities, we use Newton's method. At each step a linearized operator is defined by finite differencing the nonlinear operator, and the resultant linear system is solved using a preconditioned GMRES algorithm. The Newton's method is rarely globally convergent, and its convergence rate is generally quadratic only sufficiently close to the solution. If the initial solution is taken to be $\phi=0$, which is usually not a good approximation to the solution, the Newton's method must generally be damped, especially for large transonic problems. The most effective method we have used is based on a continuation process in which parameters are introduced to control the amount and the extent of the region of artificial viscosity. The higher dissipation is applied over a larger region during the initial Newton steps so that supersonic zones and shock positions are located fairly early in the process, even though they are quite smeared. After several Newton steps, both the amount of dissipation and the extent over which it is applied are reduced to appropriate levels. This strategy has been found to work very well. Other damping strategies based on the residual, Mach number in the field, and total temperature and pressure are also implemented and are useful in certain applications.

\section{Results}

This section presents some results obtained by applying the TRANAIR code to several practical problems of interest. The code runs on the Cray X-MP or Cray Y-MP machines with an SSD but all the results shown here are obtained on the X-MP. Results are presented for a standard test case, the ONERA M6 wing, and for two transports with nacelles, struts, and engine power. These runs have been made with up to 320,000 grid boxes and involve sparse matrix decompositions of matrices with up to 200,000 unknowns. The code is by no means optimized, but typical run times are between one and two hours for a solution converged to six to eight digit accuracy.

A standard aerodynamics test case is flow about the ONERA M6 wing at $M_{\infty}=0.84$ and $\alpha=3.06^{\circ}$. This case has an oblique supersonic to supersonic shock as well as a normal supersonic to subsonic shock. Here, TRANAIR results are compared to those obtained using FLO28 (Jameson 1983). Unusually dense grids (TRANAIR grid with 311,000 and FLO28 grid with 364,000 ) were used in both codes to capture the oblique shock as accurately as possible. Figure 4 shows a vertical cut and a waterline cut through the TRANAIR grid. Figure 5 compares surface pressures at four stations. The TRANAIR solution agrees quite well with the FLO28 solution using first-order dissipation. In this problem, both codes obtained comparable accuracy (seven digits) at comparable cost (5500 seconds for TRANAIR and 8500 seconds for FLO28).

The next case considered is flow about a 747-200 transport configuration with wing, body, struts and nacelles. The geometry description includes about 23,000 panels (see figures 6a and 7b) and is much bigger than can be handled by panel codes. The flow analysis was performed at $\alpha=2.7^{\circ}$ and $M_{\infty}=0.8$. This is approximately the 
(a)

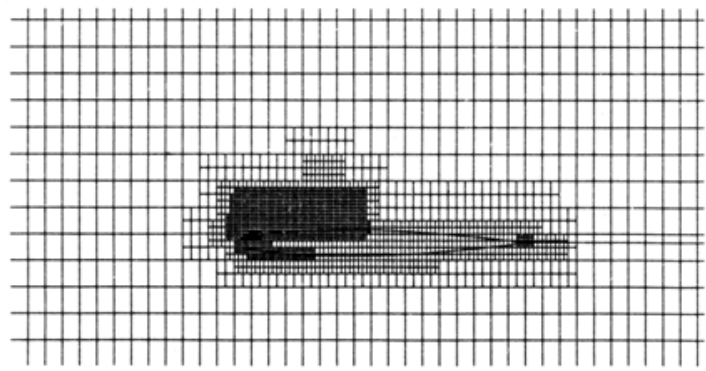

(b)

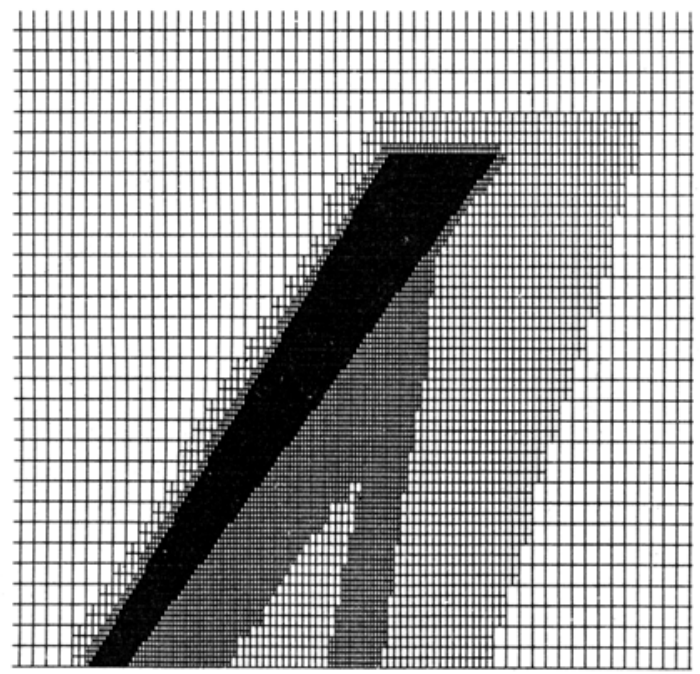

Figure 4. Two cuts through grid for ONERA M6 wing, $M_{\infty}=0.84, \alpha=3.06^{\circ}$, at $90 \%$ span (a) and at waterline (b).

largest freestream Mach number at which an inviscid solver can obtain accurate results without boundary layer coupling. The grid used for this problem consisted of approximately 219,000 finite elements. Figure 7 shows a $y z$ grid cut passing through the outboard nacelle strut and core cowl and through the prescribed wakes behind the inboard strut and nacelle. Figure 8 compares TRANAIR results with wind tunnel pressure data at four span stations of the wing. The agreement is quite reasonable. Most of the differences are seen in the upper surface pressures and are attributable to viscous boundary layer effects not currently modeled in TRANAIR. By comparing lower surface pressure profiles, one can clearly see the effects of the outbroad nacelle at the $69 \%$ span station. Near the leading edge of the wing at the $69 \%$ span station, the TRANAIR solution contains a supersonic to supersonic shock, also seen in the experimental data. The TRANAIR run took about 4,800 seconds of X-MP time. Of this, 1,900 seconds were required for overhead functions such as grid definition and stiffness matrix generation.

The last case is a transport aircraft with powered nacelles simulated by plume regions of lfigh total pressure and temperature. Figure 9 a shows the paneling for the configuration, and figure $9 \mathrm{~b}$ a typical section of the grid, which in this case contains about 230,000 boxes. Both flight idle and cruise power conditions were simulated. We present the underwing and inboard strut pressure coefficients in figures $9 \mathrm{c}$ and $d$ along with test data at the same condition. The comparison between test and theory 

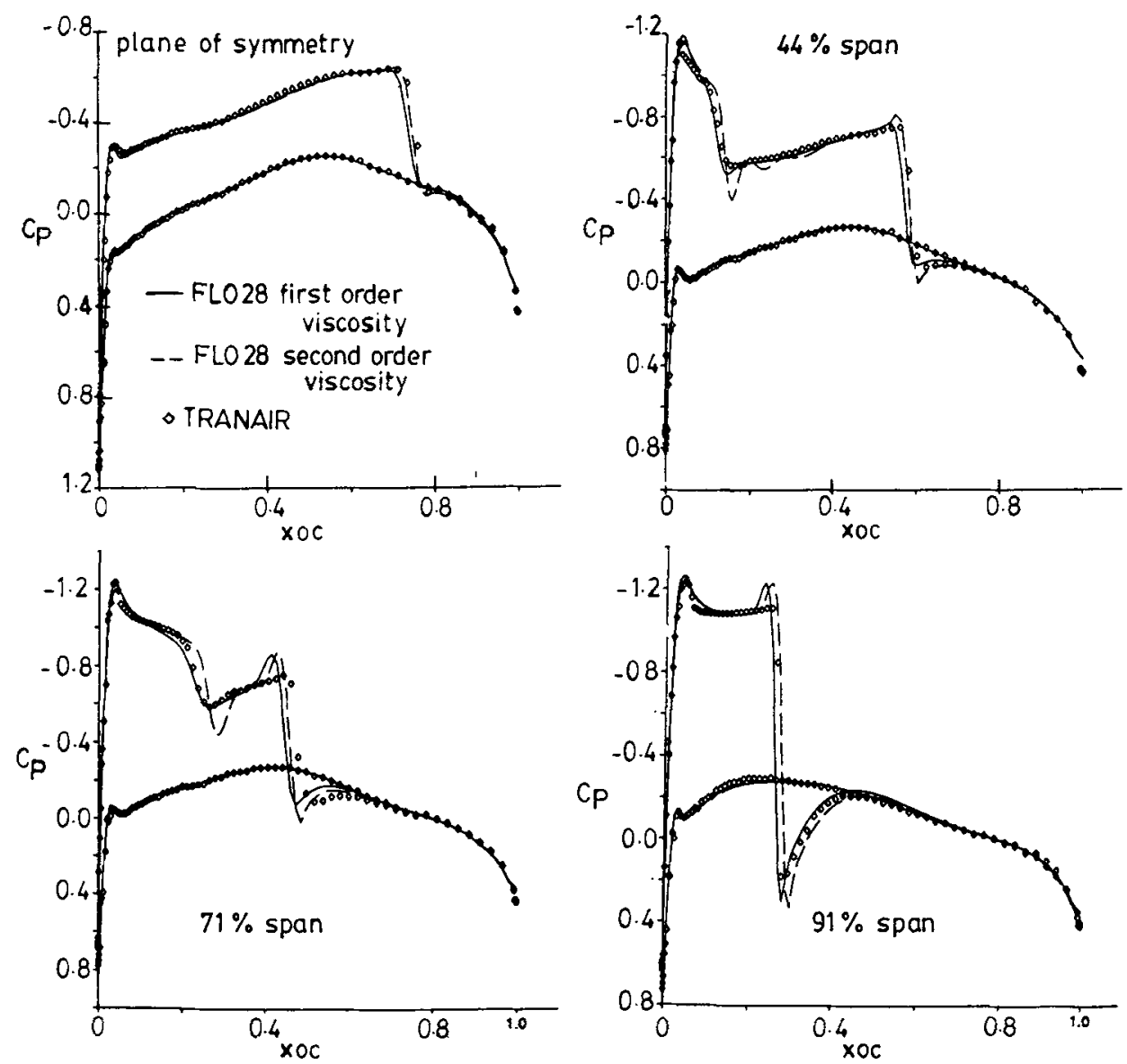

Figure 5. Comparison of surface pressures at four span stations on ONERA M6 wing, $M_{\infty}=0.84, \alpha=3.06^{\circ}$.

is remarkably good. This case demonstrates the capability of the TRANAIR code to simulate powered effects, a capability more usually associated with Euler codes.

\section{Concluding remarks}

The method described in this paper is fully implemented for aerodynamics applications in the TRANAIR computer program. The results shown in this paper demonstrate the ability of TRANAIR to handle complex geometries in transonic flow. With the ability to account for regions of different total pressure and total temperature, the code is capable of handling engine power effects accurately. The reliability, generality, efficiency and usability of TRANAIR approach those of panel codes. Run times are usually comparable to those for panel codes, even though the flow now contains shocks and other nonlinear effects.

We are currently implementing grid sequencing and adaptive grid refinement, which will further enhance the efficiency, usability and reliability of this code. The code currently lacks any modeling of viscous effects unless the externally computed 


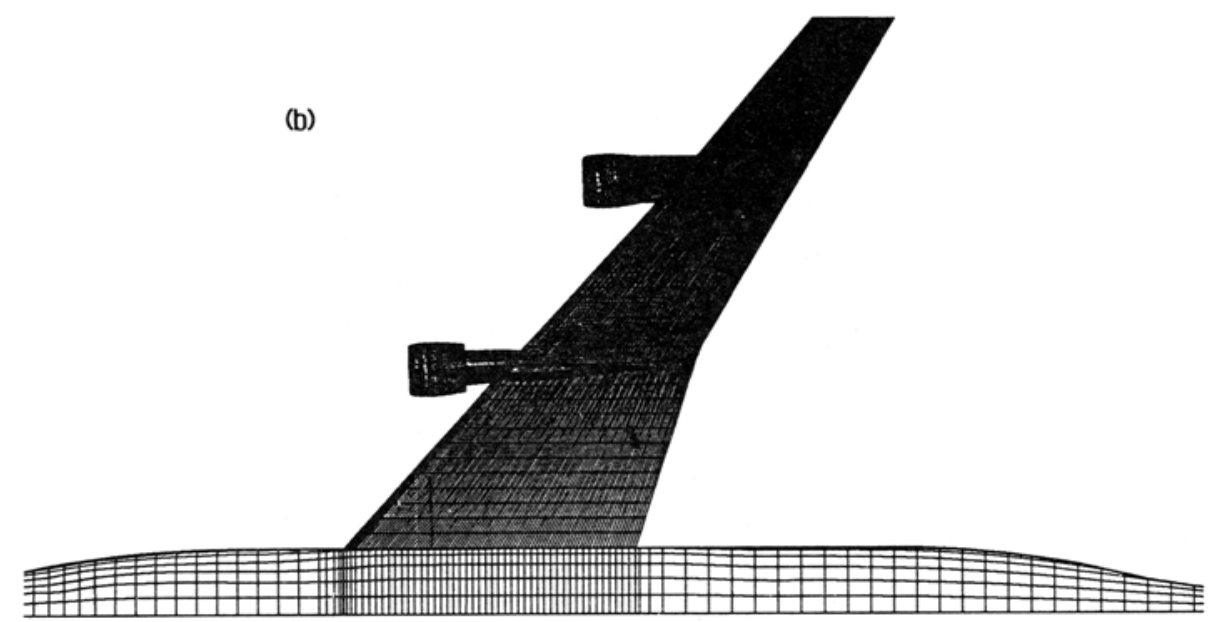

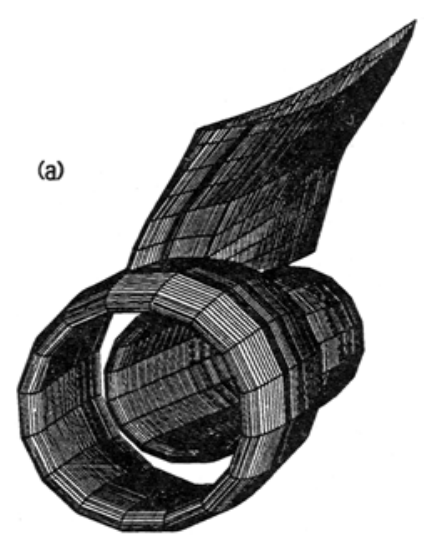

Figure 6. 747-200 transport configuration. (a) Inboard nacelle and strut, (b) top view of wing and body.

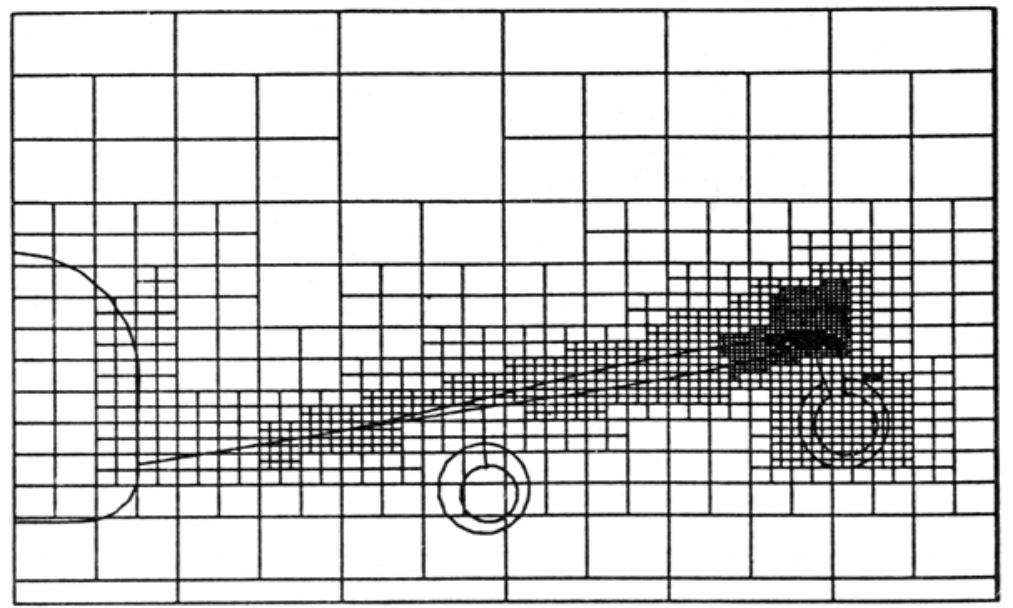

c) Constant $x$ Cut Behind Inboard Nacelle

Figure 7. A cut through"TRANAIR grid for 747-200 case. 

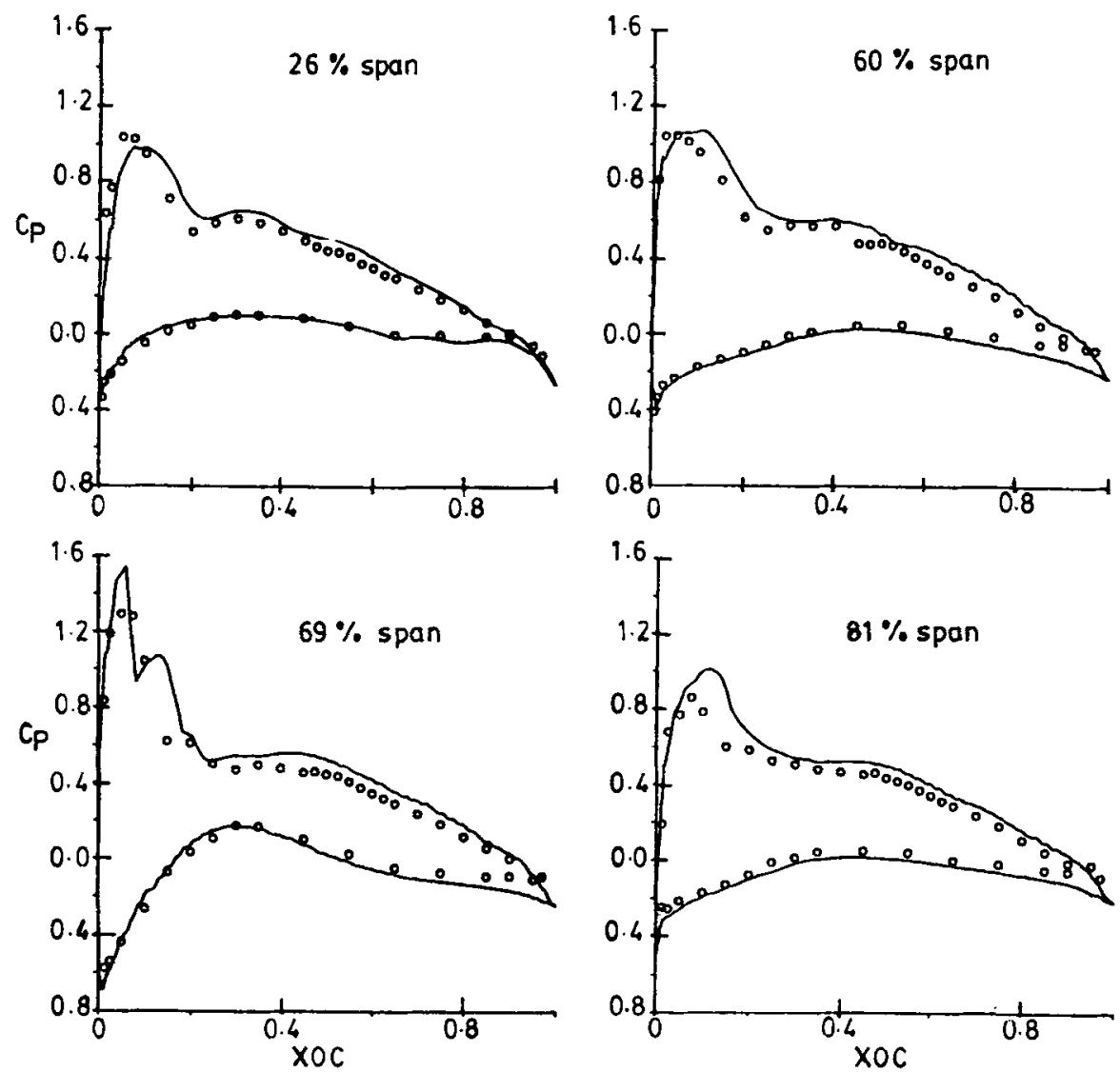

Figure 8. Wing pressures for 747-200, $M_{\infty}=0.8, \alpha=2 \cdot 7^{\circ}$.

(a)

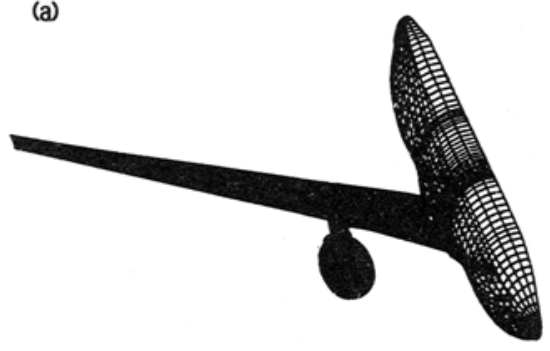

(c)

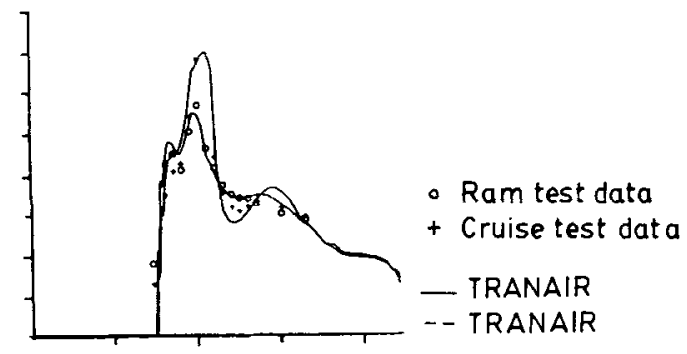

(b)

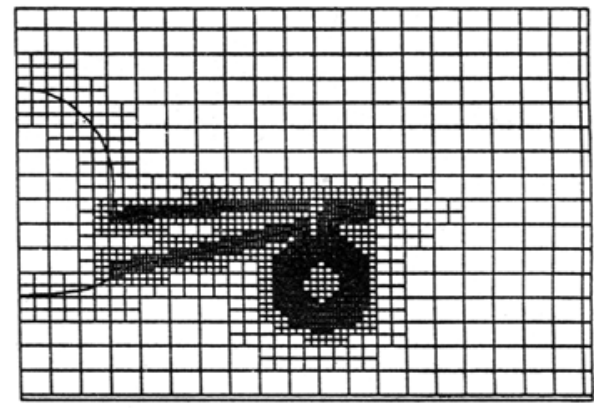

(d)

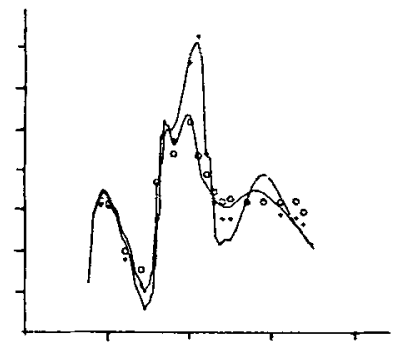

Figure 9. TRANAIR analysis of a transport with wing/body/nacelle/strut and power. (a) Surface panelling, (b) section of TRANAIR grid, (c) underwing station, (d) inboard strut station. 
transpiration boundary condition is specified. We plan to add a coupled boundary layer simulation to this code in the near future. We are also investigating a wake capturing scheme that has potential to capture sharp vortex sheets.

This work was supported in part by NASA (Contract NAS2-12513) and the IRAD funds of The Boeing Company.

\section{References}

Bateman H 1930 Irrotational motion of a compressible inviscid fluid. Proc. Natl. Acad. Sci. USA 16: 816-825

Buneman O 1971 Analytic inversion of the five-point Poisson operator. J. Comput. Phys. 8: 500-505

Bussoletti J E, Young D P, Johnson F T; Melvin R G, Samant S S, Wigton L B 1988 Application of sparse matrix solvers as effective preconditioners, Boeing Computer Services Engineering and Scientific Services Technical Report ETA-TR-99 (unpublished)

Desai S S, Rangarajan R, Singh J P, Ravichandran K S 1987 Full-potential flow computations using cartesian grids, AIAA Paper No. 87-1164, AIAA 8th Computational Fluid Dynamics Conference, Honolulu, Hawaii

Hafez M, Murman E M, South J C 1978 Artificial compressibility methods for numerical solutions of transonic full potential equations, AIAA Paper 78-1148

Jameson A 1983 Transonic flow calculations, Princeton University Department of Mechanical and Aerospace Engineering report 1651 (unpublished)

Johnson F T 1979 A general panel method for the analysis and design of arbitrary configurations in subsonic flow NASA CR-3079

Rubbert P E, Bussoletti J E, Johnson F T, Sidwell K W, Rowe W S, Samant S S, SenGupta G, Weatherill W H, Burkhart R H, Everson B L, Young D P, Woo A C 1986 A new approach to the solution of boundary value problems involving complex configuration. In Computational mechanics-Advances and trends (ed.) Ahmed $K$ Noor (New York: ASME)

Saad Y, Schultz M H 1986 GMRES: A generalized minimal residual method for solving nonsymmetric linear systems. SIAM J. Sci. Stat. Comput. 7: 856-869

Samant S S, Bussoletti J E, Johnson F T, Melvin R G, Young D P 1988 Transonic analysis of arbitrary configurations using locally refined grids. In Proceedings of the 11th International Conference on Numerical Methods in Fluid Dynamics (Berlin: Springer-Verlag)

Samet H 1984 The quadtree and related hierarchical data-structures. Comput. Surv. 16: 187-260

TRANAIR Computer Code (Theory Document), NASA Contractor's Report (unpublished)

Wedan B, South J C 1983 A method for solving the transonic full potential equation for general configurations, AIAA Paper 83-1889

Wigton L B, Yu N J, Young D P 1985 GMRES acceleration of computational fluid dynamics codes, AIAA Paper 85-1494 